\title{
Analysis of 220kV Main Transformer Screens Dendritic Discharge Fault
}

\author{
Zhan Shuguang, Li Zhi, Liu Guobin, Zhang Hua, Wang Qinghao, Zhang Lei, Pang \\ Yanjun, Zhao Chuanzong, He Lishuai, Li Jianfeng, Guan Ling, Zhou Baokun, Gao \\ Wei
}

Fushun Power Supply Company, Liaoning Electric Power Company Limited, State Grid, China, fushunpowersupply@163.com

Keyword: wet insulation; light gas; the insulating structure; screens dendritic discharge

\begin{abstract}
Through folding screen of dendritic discharge in Fushun Power Supply Company 220kV Gongnong Station, this paper folding screen in the basic process of dendritic discharge fault, argument insulation be affected with damp be affected with damp and gas are the main external cause the folding screen dendritic discharge fault; Insulation structure defects are the main internal cause folding screen dendritic discharge fault. Combined with the actual finally puts forward the measures to prevent the folding screen dendritic discharge fault.
\end{abstract}

\section{Introduction}

Folding screen dendritic discharge accident is not allow to ignore.This kind of accidents occur mostly on the three-phase transformer, unless the light gas relay action, and promptly blackout check repair. Otherwise, when a heavy gas relay action or differential protection action, the transformer will be a serious distortion, sometimes box along the bolt fracture, transformer oil leaked out, and the consequence is very serious.

Due to the transformer winding head pad and stay insulated structure exist edges, making the conductor edges in the electric field distortion. And folding screen and winding inside the protrusions (block), S bending transposition and turns uneven deformation and paperboard bending part, narrow the local oil duct, both may cause local overheating, but also intensified electric field distortion, field strength increased. Therefore, will cause the wire on the surface of the edges of the insulation oil gap occurs first discharge, then development to the folding screen board, or cause the second oil gap discharge, or along the inside surface and the interlayer sliding folding screen board flash discharge. When interlayer unclean (water or air bubbles), internal slide flash discharge is more likely to happen, finally in the plasma channel "dendritic" char is formed on the channel[1-3].

\section{The cause of the folding screen dendritic discharge}

(1)The main external cause

1) Wet insulation

The dry oil gap and cardboard folding screen structure of the electric strength is high. After the oil cut insulation board be affected with damp be affected with damp, however, its electric strength will be greatly reduced. Moisture intrusion approach generally has safe airway, hygroscopic and transformer oil pump, catheter anastomoses. When internal moisture from the way into the fuel tank, the transformer oilpaper insulation be affected with damp be affected with damp, oiled paper insulated electrical strength will be greatly reduced, trigger a folding screen dendritic discharge failure[4, 5].

2) Insulating gas

Another reason is caused by the large transformer screens dendritic discharge gas in transformer oil. Because the gas will make the discharge inception field strength greatly reduced, especially when high gas content in transformer, and moisture intrusion, because the screen board is attached to the surface moisture bubbles, or cardboard sandwich bubble, stratified and the existence of moisture whole, will be easier to form the tree discharge. 
(2)The main internal cause

The electric field strength of oiled paper composite insulation depends on the compression direction of medium thickness ratio and uniformity of the distribution of medium as a whole. If a slight lack of insulating structure in region of high electric field distribution, will accelerate the oil folding screen electric strength reduction caused by the accident.

The interphase insulating weak and unreasonable layout. In a long time in the operation of transformer, phase and relative to the average electric field intensity is different, usually white working field was higher than the average relative. In the design of transformer, if the insulation for the same level of interphase and relatively, from the structure of interphase insulation level is low; in the operation, if a phase suffered voltage effect, then the insulation level of interphase structure is lower, this is obviously unreasonable.

\section{The fault case}

On August 14, 2005 thunderstorm weather, fushun area was run in parallel with the workers and peasants become two main transformer, the load is 41000. 17 PM, no. 2 main transformer light gas or pressure relief valve "ontology" light plate, according to operating personnel immediately report schedule, at the same time check the no. 2 main transformer, the oil temperature appearance and voice no abnormalities are found, check the gas relay set gas indoor gas was about half the gas-collecting chamber of the gas. Immediately notify maintenance work area of gas sample and the sample to test by analysis.

\section{The detection results}

After the light gas signal appeared, substation maintenance personnel immediately sampled the gas and oil sample testing laboratory. Historical data are shown in Table 1.

Table 1 Historical data

\begin{tabular}{|c|c|c|c|c|c|c|c|c|c|}
\hline Date & $\mathrm{CH}_{4}$ & $\mathrm{C}_{2} \mathrm{H}_{6}$ & $\mathrm{C}_{2} \mathrm{H}_{4}$ & $\mathrm{C}_{2} \mathrm{H}_{2}$ & $\mathrm{C} 1+\mathrm{C} 2$ & $\mathrm{H}_{2}$ & $\mathrm{CO}$ & $\mathrm{CO}_{2}$ & Position \\
\hline 2002.4 .18 & 18.01 & 12.46 & 42.52 & 0.06 & 73.05 & 43 & 572 & 1971 & $\begin{array}{c}\text { The device } \\
\text { body oil }\end{array}$ \\
\hline 2002.10 .21 & 17.27 & 5.16 & 38.43 & 0 & 60.86 & 35 & 710 & 2216 & $\begin{array}{c}\text { The device } \\
\text { body oil }\end{array}$ \\
\hline 2003.4 .17 & 19.41 & 12.88 & 47.67 & 0.22 & 80.18 & 28 & 679 & 2913 & $\begin{array}{c}\text { The device } \\
\text { body oil }\end{array}$ \\
\hline 2003.10 .20 & 30.40 & 9.12 & 50.21 & 0.22 & 89.95 & 47 & 1208 & 4787 & $\begin{array}{c}\text { The device } \\
\text { body oil }\end{array}$ \\
\hline 2004.3 .3 & 30.23 & 9.43 & 44.75 & 0.27 & 84.68 & 33 & 1044 & 3984 & $\begin{array}{c}\text { The device } \\
\text { body oil }\end{array}$ \\
\hline 2004.10 .25 & 35.15 & 11.13 & 47.50 & 0.16 & 93.94 & 35 & 904 & 5201 & $\begin{array}{c}\text { The device } \\
\text { body oil }\end{array}$ \\
\hline 2005.3 .7 & 41.75 & 12.46 & 50.03 & 0.22 & 104.46 & 34 & 1471 & 4988 & $\begin{array}{c}\text { The device } \\
\text { body oil }\end{array}$ \\
\hline 2005.7 .27 & 41.82 & 11.08 & 51.44 & 0.22 & 104.56 & 27 & 1010 & 7092 & $\begin{array}{c}\text { The device } \\
\text { body oil }\end{array}$ \\
\hline 2005.8 .14 & 3017.61 & 68.17 & 675.52 & 1478.87 & 5240.17 & 23580 & 10950 & 5677 & gas \\
\hline 2005.8 .14 & 163.94 & 53.39 & 251.01 & 298.30 & 766.70 & 596 & 1273 & 7817 & $\begin{array}{c}\text { The device } \\
\text { body oil 1 }\end{array}$ \\
\hline
\end{tabular}

From the oil analysis data, hydrogen gas, acetylene, total hydrocarbon content exceeds bid badly, from the characteristics of gas, high content of acetylene and constitute the major component of total hydrocarbon, and high hydrogen content, can be judged as discharge fault. With three ratio method to102, department of high energy discharge fault (namely arc discharge). From the point of gas gas analysis data, various kinds of fault gas concentration is much higher than the oil gas concentration, failure to dissolve in oil, gas into the gas in the air, the transformer is a serious fault. From the perspective of the content of $\mathrm{CO}, \mathrm{CO}_{2}$, the fault has involved the insulation board part. Through the analysis of the above results, immediately report the bureau of the relevant departments, request the transformers stop running, maintenance checks. 
On August 15, 2005, of the transformer winding deformation, dc resistance, insulation resistance and dc leakage and dielectric loss Angle test, the results did not see abnormalities.

\section{The disintegration test conditions}

Transformer in the material on the spot check on 20 August. Check the tap-changer, lead found no abnormal situation. Check B phase high voltage coil, found the direction of high side slants of A 45 degree, by cable paper down on the number of the fourth section of the conductor surface bubbles (other parts of the folding screen to see), in the B phase by phase under the lateral upper yoke iron washer has A black board.

Opened the B phase high-voltage folding screen, the B phase to carry on the thorough inspection, found that the most layer of the cardboard, there are a large number of branches discharge one cardboard discharge severe burns. And cardboard 2 corresponding parts (bread from down to up for 5 to 10) coil block tip discharge traces, and wire and block Angle cable paper in black. The coil block point discharge plan sketch is shown in Figure 1; the coil position to screen mild discharge through the cushion block is shown in Figure 2; the insulating cardboard dendritic discharge is shown in Figure 3.

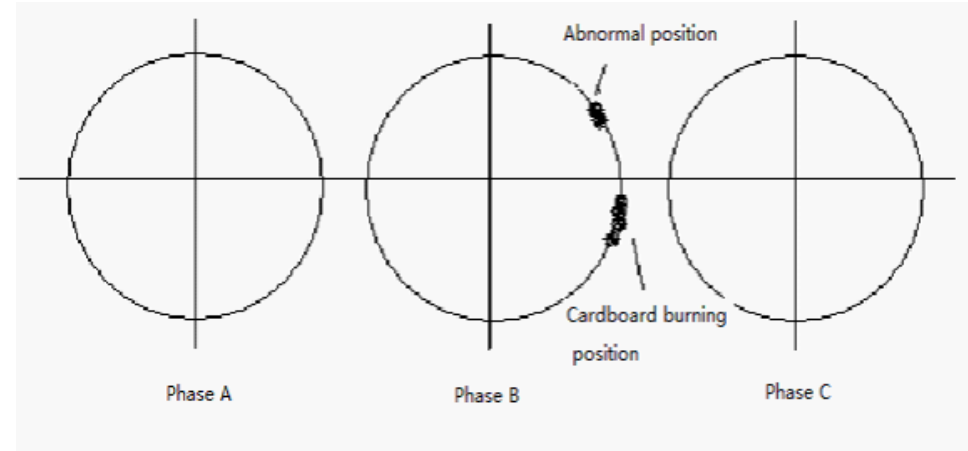

Figure 1 The coil block point discharge plan sketch

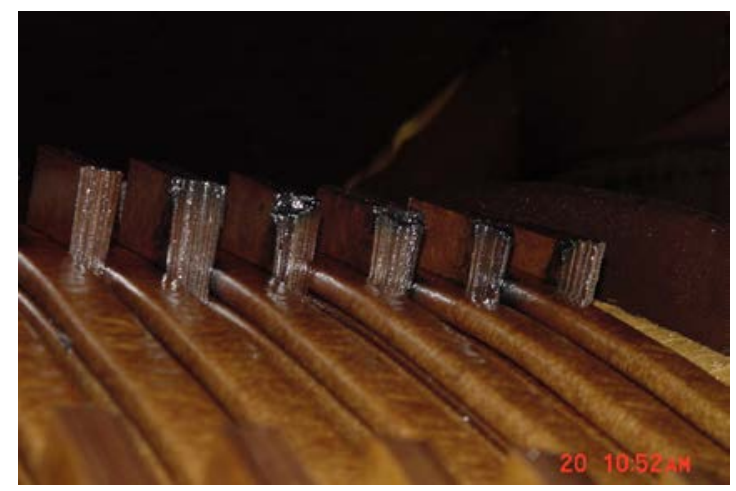

Figure 2 The coil position to screen mild discharge through the cushion block

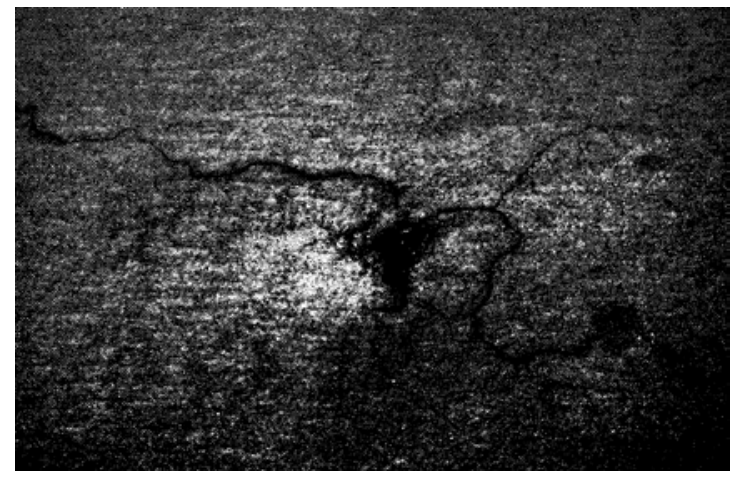

Figure 3 The insulating cardboard dendritic discharge 


\section{Conclusion}

Folding screen dendritic discharge fault is generally have precursor phenomenon, mainly manifested in the early days of dissolved gas in transformer oil hydrogen even has not reached the level to pay attention to, but usually is higher, and there are trace acetylene. Fault development to a certain stage, the light gas relay will call the police, then involved in the oil and free gas arc discharge of solid insulation fault characteristics of the gas is usually higher.

The transformer was shen changed products, 1981 chaoyang power plants will be thin insulation to the thick insulation, copper wire, aluminum wire circle, this is transformer folding screen dendritic discharge is the internal cause of the accident.

\section{Reference}

[1] Dong Qiguo. Power transformer fault and diagnosis. China Electric Power Press, 2000

[2] Chengdaxian. Mechanical Design Handbook. Chemical Industry Press, 1999.

[3] Zhonghongbi, Gaozhan-bang. Power transformer maintenance and testing manual. China Electric Force Press, 1999.

[4] Han Aizhi. The Simple Method of Judging Transformer Winding Deformation. Transformer, 2003, 5(4): 14-16

[5] Chen Tianxiang, Wang Yinzhong. Electrical Test. China Power Press, 2010 